\title{
Amplitude-Dependent Dynamical Behavior of Poly(methyl methacrylate)
}

\author{
F. Povolo ${ }^{\dagger}$ and S. N. GoYANES \\ Universidad de Buenos Aires, Facultad de Ciencias Exactas y Naturales, Departamento de Física, \\ Pabellón 1, Ciudad Universitaria, 1428 Buenos Aires, Argentina
}

(Received January 12, 1994)

\begin{abstract}
Some results on the amplitude-dependent dynamical behavior of poly(methyl methacrylate) are presented. Both the loss tangent (internal friction) and the storage Young's modulus are strain-amplitude-dependent, when measured between room temperature and approximately $400 \mathrm{~K}$, at a frequency of the order of $50 \mathrm{kHz}$. The data are interpreted in terms of an hyperbolic sine stress dependence of the strain rate, including an effective stress. The activation volume was found to depend on the temperature, with values much larger than those obtained through the usual mechanical tests (tensile, creep, or stress relaxation).

KEY WORDS Poly(methyl methacrylate) / Dynamic Mechanical Behavior /

Loss Tangent / Storage Young's Modulus / Strain Amplitude-Dependent

Damping and Modulus /
\end{abstract}

Several data on the dynamic mechanical behavior of poly(methyl methacrylate), (PMMA) have been reported, and several reviews have been written on relaxation processes in amorphous glassy polymers, including PMMA. ${ }^{1-3}$ The dynamic mechanical behavior data (storage and loss moduli, loss tangent) have been interpreted mainly in terms of linear viscoelasticity, appropriate for small strains, and the study of non-linear viscoelastic and plastic phenomena has been restricted mainly to large deformations, like those obtained in stressstrain curves and creep (ref 3, Chapter 10).

Gilbert, et al. ${ }^{4}$ have constructed modulusmechanism maps for amorphous PMMA and polystyrene. Four regimes were identified: the glassy regime, the viscoelastic regime, the rubbery regime and the regime of viscous flow (melting), truncated by decomposition. Constitutive laws were given for each regime which were used to construct diagrams which related the time and temperature dependent modulus, $E(t, T)$, to the temperature and loading time (or the frequency). The diagrams summarize the small-strains (linear) mechanical behavior of the polymers and assume linear viscoelasticity.

Bauwens-Crowet ${ }^{5}$ has studied the compression yield behavior of poly(methyl methacrylate) over a wide range of temperatures and strain rates. He interpreted the yield data using a modification of Ree-Eyring theory and correlated the yield behavior with the $\beta$ mechanical loss peak observed in damping tests. A distribution of relaxation times and linear viscoelasticity was assumed for the $\beta$ peak and non-linear behavior was assumed for the strain-rate dependence of the yield stress.

Amplitude-dependence of the dynamic properties have been observed in rubber compounds. ${ }^{6}$ These effects were observed both in the dynamic moduli and the loss tangent, for

† Comisión Nacional de Energía Atómica, Departamento de Materiales, Av del Libertador 8250, 1429 Buenos Aires, Argentina. 
strain amplitudes above $1 \times 10^{-3}$. Wanaka and Miller ${ }^{7}$ have pointed out that non-linearity or strain-sensitivity of the dynamic moduli should be present, in homogeneous, amorphous polymers, at dynamic strains higher than about $1 \times 10^{-3}$. Experimental data have been presented, however, only for rubber compounds.

It is the purpose of this paper to present some results on the amplitude-dependence of the dynamic moduli and the damping of commercial PMMA, for strain amplitudes between $1 \times 10^{-7}$ and $1 \times 10^{-5}$. Both the strain-amplitude and the temperature dependence of the dynamic response will be considered, from room temperature up to about $400 \mathrm{~K}$ and at a frequency of the order of $50 \mathrm{kHz}$.

\section{EXPERIMENTAL}

The measuring device involves the excitation of the specimen by piezoelectric crystals. ${ }^{8} \mathrm{~A}$ common variation of this general technique, first introduced by Marx, ${ }^{9}$ is shown in Figure 1. The Marx oscillator consists of three basic components (a drive crystal, a gauge crystal and the specimen) each of which is cut to resonate at a specified frequency (about $50 \mathrm{kHz}$ in our case) in longitudinal vibrations. Often a dummy rod with a length equal to an integral number of half-wavelengths of the specified frequency is inserted between the specimen and the driver crystal so that the temperature of the specimen may be changed while the crystals

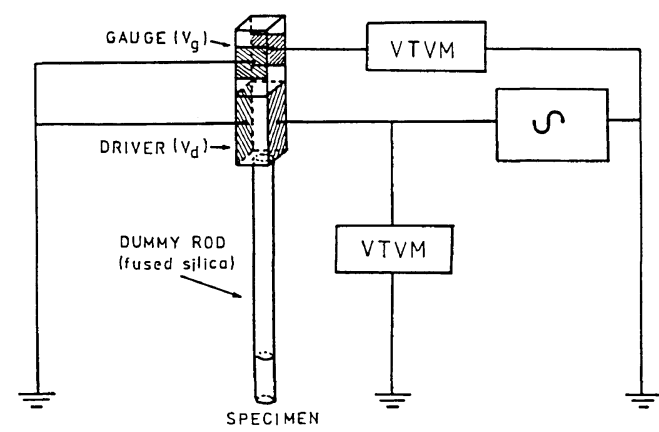

Figure 1. Marx composite oscillator. are maintained at room temperature.

The main features of the Marx system is that the damping, the storage modulus and strain amplitude can be determined from the measurement of three quantities: the drive voltage, $V_{\mathrm{d}}$, the gauge voltage, $V_{\mathrm{g}}$, and the resonant frequency, $f_{\mathrm{r}}$. The damping of the system, $\delta_{\mathrm{t}}$, is given by ${ }^{9,10}$

$$
\delta_{\mathrm{t}}=\alpha\left(V_{\mathrm{d}} / V_{\mathrm{g}}\right)
$$

where $\alpha$, the proportionality constant, may be calculated or determined experimentally from the width of the resonance curve in the gauge circuit. ${ }^{9,10}$

The strain amplitude of all components of the system is a linear function of the gauge voltage. The strain, $\varepsilon_{0}$, in the specimen is

$$
\varepsilon_{0}=\beta V_{\mathrm{g}}
$$

where $\beta$ depends on the mechanical overtone and can be determined experimentally. ${ }^{9,10}$

The damping of the specimen, $\delta_{\mathrm{s}}$, is obtained from the total damping and the damping of the crystals-dummy composite, $\delta_{c}$, by the relationship

$$
m_{\mathrm{t}} \delta_{\mathrm{t}}=m_{\mathrm{s}} \delta_{\mathrm{s}}+m_{\mathrm{c}} \delta_{\mathrm{c}}
$$

Here $m_{\mathrm{s}}, m_{\mathrm{c}}, m_{t}$ are the masses of the specimen, the crystals-dummy composite, and the total mass $m_{\mathrm{s}}+m_{\mathrm{c}}$ of the system, respectively.

In longitudinal vibrations, the storage Young's modulus of the specimen, $E_{\mathrm{s}}^{\prime}$, is given by ${ }^{11}$

$$
2 f_{\mathrm{s}} l_{\mathrm{s}}=\left(E_{\mathrm{s}}^{\prime} / \rho_{\mathrm{s}}\right)^{1 / 2}\left(1-\delta_{\mathrm{s}}^{2} / 8\right)
$$

which, for low damping reduces to the expression

$$
E_{\mathrm{s}}^{\prime}=\rho_{\mathrm{s}}\left(2 l_{\mathrm{s}} f_{\mathrm{s}}\right)^{2}
$$

where $\rho_{\mathrm{s}}$ is the density, $l_{\mathrm{s}}$ the length and $f_{\mathrm{s}}$ the resonant frequency of the specimen.

The resonant frequency of the specimen can be obtained by using the relationship ${ }^{10}$

$$
m_{\mathrm{t}} f_{\mathrm{t}}^{2}=m_{\mathrm{s}} f_{\mathrm{s}}^{2}+m_{\mathrm{c}} f_{\mathrm{c}}^{2}
$$

where $f_{\mathrm{c}}$ and $f_{\mathrm{t}}$ are the resonant frequencies of 
the composite and the system, respectively.

A fused silica dummy rod was used for the measurements and the dummy rod and the specimen were located into a furnace, so that the temperature of the specimen could be changed. In order to ensure homogeneity of temperature in the specimen, a dummy specimen, also attached to a fused silica rod, was located near the specimen. Two thermocouples introduced near the ends of the dummy specimen were used to ensure homogeneity of temperature and a third thermocouple, located near the specimen, was used also to sense the temperature. With this arrangements it was possible to ensure homogeneity of temperature in the specimen, both axially and radially. All the measurements were performed mainly on heating and after a complete stabilization of the selected temperature, which was kept stable within $\pm 0.5 \mathrm{~K}$.

The material used was commercial PMMA, named Vicalon, supplied by a local firm. This material was fabricated by molding according to ASTM D788-84 standard. According to the supplier, the material had a content of free monomers of methyl methacrlylate lower than $1 \%$. Specimens in the form of rods with $3 \mathrm{~mm}$ in diameter and of the order of $18 \mathrm{~mm}$ in length were used.

\section{RESULTS}

Figure 2 shows the damping and Figure 3 the storage Young's modulus as a function of the strain amplitude, for various temperatures. It is seen that both the damping and the modulus are amplitude independent at very low strains and both become amplitude dependent at higher strains. Then, both quantities are divided in two parts: one amplitude independent and the other one amplitude dependent, that is,

$$
\delta_{\mathrm{s}}=\delta_{\mathrm{I}}+\delta_{\mathrm{H}}
$$

where $\delta_{\mathrm{I}}$ is the amplitude independent part of the damping and $\delta_{\mathrm{H}}$ the amplitude dependent

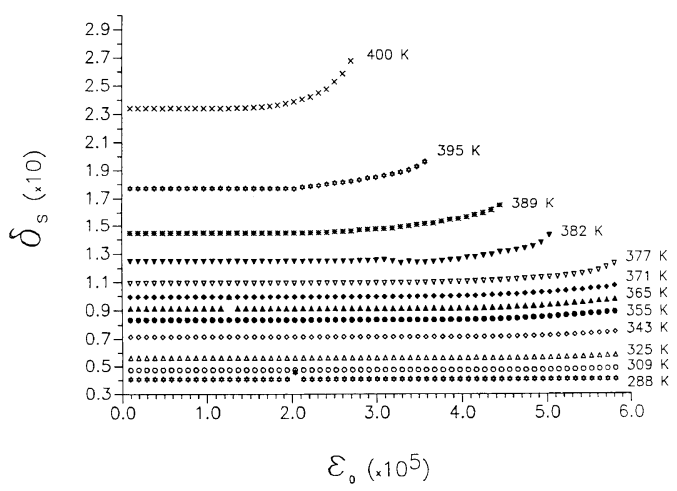

Figure 2. Damping (loss tangent) of PMMA as a function of strain amplitude at various temperatures.

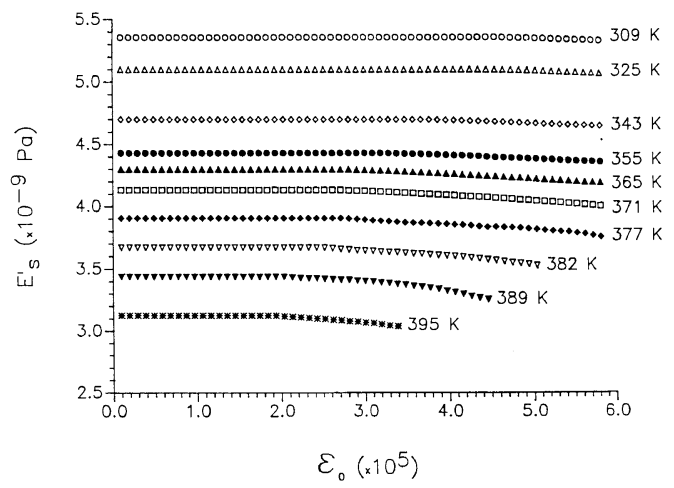

Figure 3. Storage Young's modulus of PMMA as a function of strain amplitude at various temperatures.

part. A similar expression was used for the modulus. A procedure described elsewhere ${ }^{12}$ was used to separate, with great accuracy, each contribution.

Figure 4 illustrates the amplitude dependent part of the damping and Figure 5 the amplitude dependent part of the modulus, as a function of the strain amplitude and for various temperatures. The amplitude dependent part of the modulus, or modulus defect, is defined as

$$
\Delta E^{\prime} / E^{\prime}=\left(E_{\mathrm{os}}^{\prime}-E_{\mathrm{s}}^{\prime}\right) / E_{\mathrm{os}}^{\prime}=\left(f_{\mathrm{os}}^{2}-f_{\mathrm{s}}^{2}\right) / f_{\mathrm{os}}^{2}
$$

where $f_{\text {os }}$ is the resonant frequency of the specimen at low strain amplitudes and $f_{\mathrm{s}}$ the resonant frequency at any strain amplitude.

Figure 6 shows the amplitude indepen- 


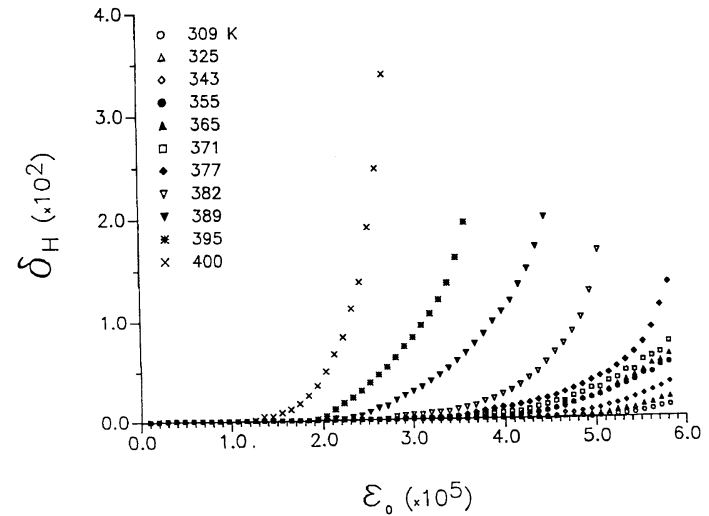

Figure 4. Amplitude dependent damping of PMMA as a function of strain amplitude at various temperatures.

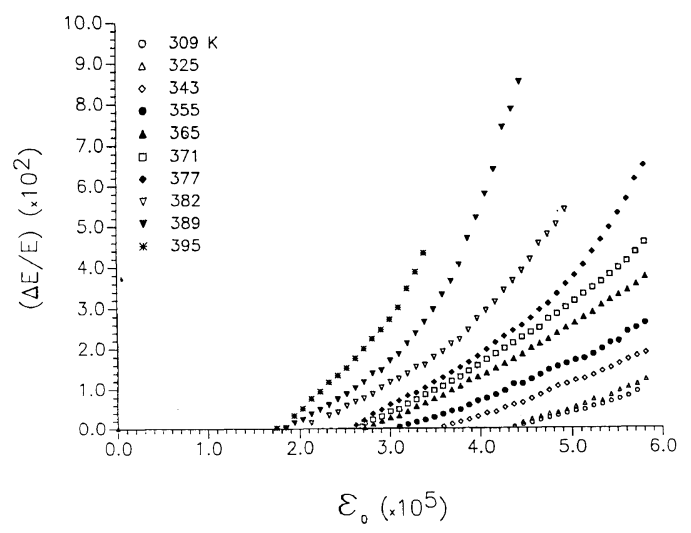

Figure 5. Amplitude dependent modulus defect of PMMA as a function of strain amplitude at various temperatures.

dent damping, $\delta_{\mathrm{I}}$, the amplitude independent modulus, $E_{\mathrm{I}}^{\prime}$, and the critical strain, $\varepsilon_{\mathrm{c}}$, at which amplitude dependence starts, both in the damping and in the modulus, as a function of temperature.

It should be pointed out that when the damping or the modulus defect are amplitude dependent a correction must be made to the measured curves. In effect, on comparing the experimental results with the theoretical models it is assumed that the stresses applied to the specimen are homogeneous. This is not the case when the damping is measured by resonant techniques, where non-homogeneous

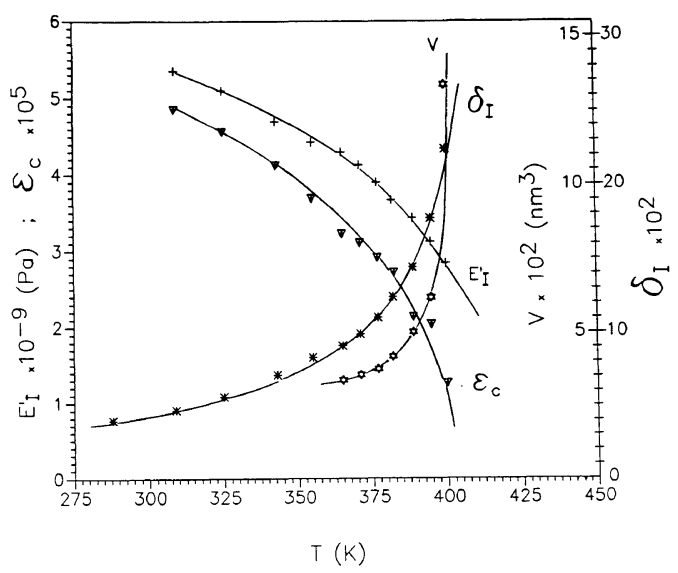

Figure 6. Amplitude independent damping, $\delta_{1}$, amplitude independent storage modulus, $E_{\mathrm{I}}^{\prime}$, critical strain for the beginning of the amplitude dependence, $\varepsilon_{\mathrm{c}}$, and activation volume, $v$, for PMMA as a function of temperature.

stresses are applied to the specimens. A similar situation is encountered for measurements performed with a torsion pendulum. Procedures have been developed ${ }^{12-16}$ to reduce experimental curves of damping and modulus defect under non-uniform stress versus maximum strain amplitude to those intrinsic or true values that would be measured if the stress (or strain) distribution in the specimen were uniform. In the case of longitudinal excitations, which are considered in the paper, the intrinsic damping or modulus defect is given by

$$
A\left(\varepsilon_{1}\right)=\frac{1}{2 \varepsilon_{1}^{2}} \frac{\mathrm{d}}{\mathrm{d} \varepsilon_{1}} \int_{0}^{\varepsilon_{1}}\left\{e_{0}^{3} A_{\mathrm{m}}\left(\varepsilon_{0}\right) / \sqrt{\varepsilon_{1}^{2}-\varepsilon_{0}^{2}}\right\} \mathrm{d} \varepsilon_{0}
$$

where $A_{\mathrm{m}}\left(\varepsilon_{0}\right)$ is the measured damping or modulus defect at a maximum strain amplitude $\varepsilon_{0}$ and $A\left(\varepsilon_{1}\right)$ is the intrinsic value at an uniform strain $\varepsilon_{1}$.

Finally, no substantial differences in the damping behavior was encountered during measurements performed after annealing the specimen for two hours at $413 \mathrm{~K}$, previous to the dynamical tests. In effect, practically the same amplitude-dependent damping was ob- 
served and only slight changes in $\delta_{\mathrm{I}}$ were obtained. These results together with observations performed in pre-deformed specimens will be presented in another publication.

\section{DISCUSSION}

\section{Amplitude-Dependent Damping}

The strain rate, $\dot{\varepsilon}$, under an applied stress, $\sigma$, is given in terms of the Ree-Eyring equation by

$$
\dot{\varepsilon}=\dot{\varepsilon}^{*} \exp (-\Delta H / k T) \sinh \left(\bar{\sigma}_{v} / 2 k T\right)
$$

where $\Delta H$ and $v$ are the activation enthalpy and activation volume, respectively, $\dot{\varepsilon}^{*}$ is the pre-exponential factor, $k$ and $T$ are Boltzmann's constant and the absolute temperature, respectively. $\bar{\sigma}=\sigma-\sigma_{\mathrm{i}}$, where $\sigma_{\mathrm{i}}$ is an internal stress.

It is possible to calculate the damping by making an analysis similar to the one proposed by Peguin, et $a{ }^{17}{ }^{17}$ In fact, at constant temperature eq. 10 can be written as

$$
\dot{\varepsilon}=A_{1} \sinh \left(B_{1} \bar{\sigma}\right)
$$

with

$$
\begin{gathered}
\sigma_{\mathrm{i}}=E_{1}^{\prime} \varepsilon_{\mathrm{c}} \\
A_{1}=\dot{\varepsilon}^{*} \exp (-\Delta H / k T)
\end{gathered}
$$

and

$$
B_{1}=v / 2 k T
$$

For a sinusoidal stress of amplitude $\sigma=$ $\sigma_{0} \sin \omega t$, the amplitude-dependent damping, given by

$$
\delta_{\mathrm{Hc}}=\left(E_{\mathrm{I}}^{\prime} / \sigma_{0}^{2}\right) \int_{0}^{2 \pi / \omega} \sigma \dot{\varepsilon} \mathrm{d} t
$$

can be calculated as

$$
\delta_{\mathrm{Hc}}=\left(A_{2} / \sigma_{0}\right)\left[I_{1}\left(B_{1} \sigma_{0}\right)-I_{1}\left(B_{1} \sigma_{\mathrm{i}}\right)\right]
$$

where

$$
A_{2}=2 A_{1} E_{\mathrm{I}}^{\prime} \cosh \left(B_{1} \sigma_{\mathrm{i}}\right) / \omega
$$

$I_{1}$ is the integral form of the modified Bessel function of the first order and $\delta_{\mathrm{Hc}}$ indicates the intrinsic amplitude-dependent damping.

In terms of the strain, finally eq 16 can be written as

$$
\begin{aligned}
\delta_{\mathrm{Hc}}= & \left(2 \dot{\varepsilon}^{*} / \varepsilon_{0} \omega\right) \exp (-\Delta H / k T) \\
& \times \cosh \left(v E_{\mathrm{I}}^{\prime} \varepsilon_{\mathrm{c}} / 2 k T\right)\left[I_{1}\left(v E_{\mathrm{I}}^{\prime} \varepsilon_{0} / 2 k T\right)\right. \\
& \left.-I_{\mathrm{I}}\left(v E_{\mathrm{I}}^{\prime} \varepsilon_{\mathrm{c}} / 2 k T\right)\right]
\end{aligned}
$$

In the derivation of eq 18 it was assumed that the storage modulus does not depend on the strain amplitude. This assumption should not alter substantially the results since $E_{\mathrm{s}}^{\prime}$ changes only slightly with $\varepsilon_{0}$.

The curves of Figure 4, corrected for inhomogeneous strains according to eq 9 , were fitted to the equation

$$
\varepsilon_{0} \delta_{\mathrm{Hc}}=A\left[I_{1}\left(a \varepsilon_{0}\right)-I_{1}\left(a \varepsilon_{\mathrm{c}}\right)\right]
$$

with $\varepsilon_{\mathrm{c}}$ given, at each temperature, by Figure 6 , obtaining a pair of values

$$
A=\left[2 \dot{\varepsilon}^{*} \exp (-\Delta H / k T) / \omega\right] \cosh \left(a \varepsilon_{\mathrm{c}}\right)
$$

and

$$
a=v E_{\mathbf{I}}^{\prime} / k T
$$

at each temperature. The quality of the fitting of the experimental data to eq 19 is illustrated in Figure 7.

Once $\mathrm{A}$ and $\mathrm{a}$ are known, it is possible to

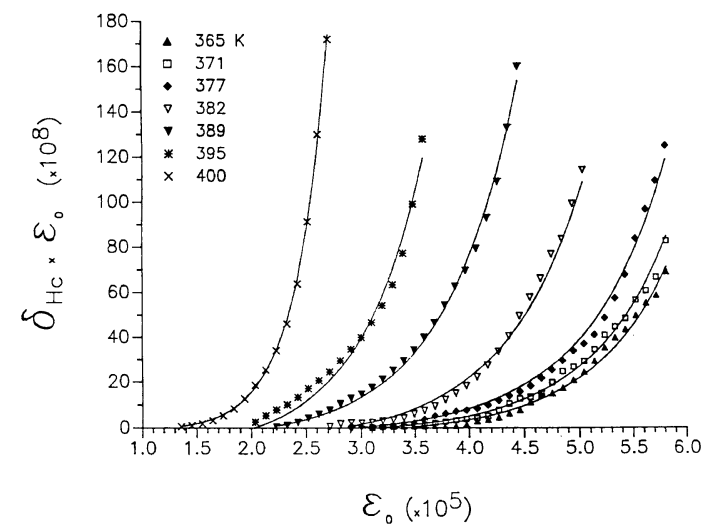

Figure 7. Comparison between the values given by eq $19^{19}$ (full curves) and the experimental data, for the intrinsic amplitude-dependent damping. 
calculate $v, \varepsilon^{*}$, and $\Delta H$ as a function of temperature, taking into account the values of $E_{\mathrm{I}}^{\prime}$ and $\varepsilon_{\mathrm{c}}$ given in Figure 6 . This procedure leads to

$$
\Delta H=114 \mathrm{k} \mathrm{J} \mathrm{mol}^{-1}
$$

and

$$
\dot{\varepsilon}^{*}=9.8 \times 10^{10} \mathrm{~s}^{-1}
$$

\section{Amplitude Independent Damping}

The amplitude independent damping, measured at $50 \mathrm{kHz}$ and shown in Figure 6, can be described by the expression

$$
\delta_{\mathrm{I}}=2.6 \times 10^{-2}+2.91 /(413-T)
$$

Then, $\delta_{1}$ does not increase exponentially with the reciprocal of the temperature showing that it does not represent the left side of a traditional relaxation peak. A similar behavior, in the sense that $\delta_{\mathrm{I}}$ also increased monotonously with temperature, was reported by Heijboer ${ }^{18}$ at frequencies of the order of $2 \mathrm{kHz}$. No information was given by the author, however, on the amplitude of the strain applied during the measurements. In any case, also the total damping at $50 \mathrm{kHz}$ increases steadily with temperature. Wunderlich ${ }^{19}$ reported, at $298 \mathrm{~K}$, a dissipation factor of $5.5 \times 10^{-2}$ at $1 \mathrm{kHz}$ and $1.4 \times 10^{-2}$ at $1 \mathrm{MHz}$, respectively. From Figure 2 , the dissipation factor at $50 \mathrm{kHz}$ and at this temperature is of the order of $4 \times 10^{-2}$. Heijboer reported a value of the order of $5 \times 10^{-2}$ at $2 \mathrm{kHz}$. It is clear that more work is needed to determine the physical mechanisms controlling $\delta_{\mathrm{I}}$, which represents the real linear viscoelastic response of the material.

\section{Storage Modulus}

$E_{\mathrm{I}}^{\prime}$ shows a temperature dependence of the type of eq 24 , with a similar critical temperature. Wunderlich ${ }^{19}$ reported a value of 3.3 GPa for the modulus of elasticity of PMMA at $298 \mathrm{~K}$. According to Figure 6, however, the storage Young's modulus at $298 \mathrm{~K}$ is 5.44 $\mathrm{GPa}, \mathrm{a}$ value which is substantially higher. No information was given by Wunderlich on the frequency of the measurement.

A value of $5.44 \mathrm{GPa}$ FOR $E^{\prime}$ leads to $G^{\prime} \simeq E^{\prime} / 3=1.81 \mathrm{GPa}$ for PMMA, at $295 \mathrm{~K}$ and $50 \mathrm{kHz}$. According to Muzeau and Perez ${ }^{20}$ the storage shear modulus at $300 \mathrm{~K}$ is $0.65 \mathrm{GPa}$ at $1 \mathrm{~Hz}$, as obtained with a torsion pendulum. Houssey et al. ${ }^{21}$ reported a value of $0.016 \mathrm{GPa}$ for $G^{\prime}$ at $1.7 \mathrm{~Hz}$ and $T=300 \mathrm{~K}$. According to Lefebvre and Escaig ${ }^{22} G^{\prime}=1.63 \mathrm{GPa}$ at $7.8 \mathrm{~Hz}$ and at the same temperature. Finally, according to $\operatorname{Read}^{23} G^{\prime}=1.2 \mathrm{GPa}$ and $1.6 \mathrm{GPa}$ at 1 and $40 \mathrm{~Hz}$, respectively, at room temperature. The value reported by Reed, however, were calculated from Young's modulus and Poisson ratio obtained by tensile non-resonance data. The results just described show that there are important disagreements in the literature about the moduli of PMMA and on their frequency dependence. Further work is needed in this field.

\section{Activation Volume}

An interesting point to be discussed refers to the temperature dependence of the activation volume, indicated in Figure 6. This temperature dependence can be very well described by the equation

$$
v=221+3980 /(404-T) \mathrm{nm}^{3}
$$

indicating that the temperature dependence of $v$ might be related to the glass transition temperature. In effect, the glass transition temperature of PMMA determined by dilatometric methods is of the order of $378 \mathrm{~K},{ }^{19}$ varies between 266 and $399 \mathrm{~K}$ according to Van Krevelen, ${ }^{24}$ and it is of the order of $388 \mathrm{~K}$ according to Ferry. ${ }^{\text {1a }}$ On considering WilliamsLandel-Ferry equation, ${ }^{1 b}$ the $\alpha$ transition will occur, at a frequency of $50 \mathrm{kHz}$, at the temperature given by

$$
T_{\mathrm{g}}^{\prime}=388+C_{2} \log f /\left(C_{1}-\log f\right)
$$

with $f=5 \times 10^{5} \mathrm{~s}^{-1}, C_{1}=32.2$ and $C_{2}=80 \mathrm{~K}$, leading to 


$$
T_{\mathrm{g}}^{\prime}=403 \mathrm{~K}
$$

This temperature is quite similar to the critical temperature of $404 \mathrm{~K}$ in eq 25 and not too far from the critical temperature of $413 \mathrm{~K}$ in eq 24. In any case, the analysis of the temperature dependence of both $v$ and $\delta_{\mathrm{I}}$ should be considered with caution due to the narrow range in temperatures explored. Also the Williams-Landel-Ferry equation represents only an approximation.

It is interesting to point out that Cavrot et $a l .{ }^{25}$ found a temperature dependent activation volume for the thermally activated deformation of glassy polystyrene. In fact, $v$ increases very rapidly above about $300 \mathrm{~K}$ and its temperature dependence can be very well described by the expression

$$
v=0.33+27.2 /(385-T) \mathrm{nm}^{3}
$$

The critical temperature of $385 \mathrm{~K}$ is very close to the glass transition temperature of polystyrene which, according to Van Krevelen ${ }^{24}$ varies between 353 and $373 \mathrm{~K}$.

The measured activation volume, shown as a function of temperature in Figure 6, varies between 300 and $1300 \mathrm{~nm}^{3}$. These values are much higher than those normally reported for glassy polymers, ${ }^{25-32}$ which are of the order of $1 \mathrm{~nm}^{3}$. These activation volumes, however, were obtained at much higher stresses, through tensile, stress-relaxation, or creep experiments. Pink, ${ }^{29}$ for example, has shown that the activation volume for PVC decreases as the applied stress increases, for stresses between 20 and $120 \mathrm{MPa}$. In our case, the maximum stress used is of the order of $0.25 \mathrm{MPa}$, at room temperature, which is much lower than those normally used with other techniques. For example, the minimum compression yield stress measured at room temperature by BauwensCrowet $^{5}$ in PMMA was of the order of 88 $\mathrm{MPa}$. Then, the activation work in our case is of the order of $\sigma v=0.25 \times 300=75 \mathrm{MPa} \mathrm{nm}$ and for the data of Bawens-Crowet is $\sigma v=88 \times 0.38=33 \mathrm{MPa} \mathrm{nm}^{3}$. It is seen that the activation works are of the same order of magnitude in both cases.

In the high frequency experiments used in this work the activation volume must be much higher than in quasistatic conditions, in order that the sinh term of eq 10 plays a role in the strain rate. The stresses applied at high frequency are very small and are under the limit of detection of quasistatic conditions. Furthermore, also the strain rates are much lower. In effect, eq 10 , with the values of $\Delta H$ and $\dot{\varepsilon}^{*}$ given by eq 22 and 23 , leads to a maximum strain rate of the order of $10^{-6} \mathrm{~s}^{-1}$ at $300 \mathrm{~K}$. This value is at least an order of magnitude lower than the minimum strain rate used in the reported tensile values. It is difficult at this point to explain the physical mechanisms leading to the high activation volumes obtained experimentally in this work. It is clear that several monomers moving cooperatively are involved in the relaxation process, since the activation enthalpy, given by eq 22 , is quite similar to the one obtained during measurements of the yield point. ${ }^{5,33}$

\section{Internal Stress}

The internal stresses, given by eq 12 with $\varepsilon_{\mathrm{c}}$ and $E_{\mathrm{I}}^{\prime}$ given by Figure 6 , as obtained at high frequencies are much lower than the values

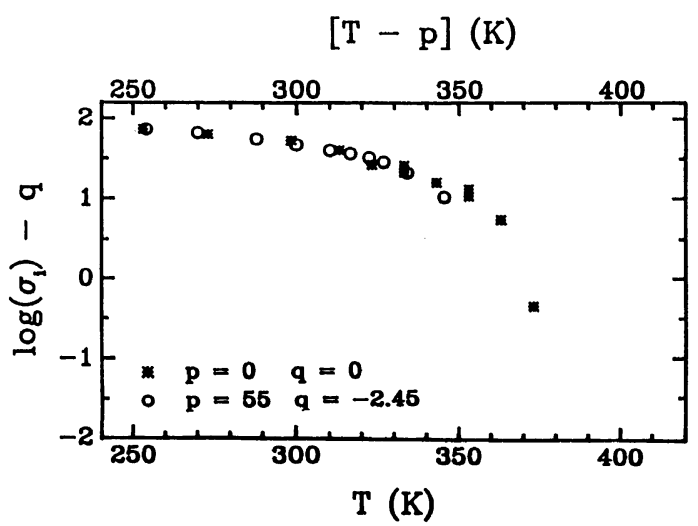

Figure 8. Temperature dependence of the internal stress measured through the yield point $(*)$ and using the high frequency technique (O). $p$ and $q$ are the horizontal and vertical shift, respectively, to superpose the dynamical data to the quasistatic ones. 
measured during tensile or compression tests. ${ }^{33}$ As shown in Figur 8, all the data obtained under quasistatic and dynamic conditions can be superposed to form an unique curve. A horizontal shift of $-55 \mathrm{~K}$ and a vertical shift of 2.45 decades must be applied to the data at high frequencies with respect to the values obtained during tensile or compression tests. In other words, the material behaves at high frequencies as if it were harder at equivalent temperatures. Lower values of $\sigma_{\mathrm{i}}$ are obtained at high frequencies due to the fact that the sensitivity of technique is much higher. It is evident that more theoretical and experimental work is needed to establish a clear correlation between the high frequency and the quasistatic results. Even the mechanisms acting under quasistatic conditions are not clearly understood.

\section{CONCLUSIONS}

It has been shown that the dynamic mechanical behavior of PMMA is temperature and strain-amplitude dependent. The amplitude dependent damping can be described by assuming a hyperbolic sine stress dependence of the strain rate, indicating non-linear viscoelastic behavior. Much larger activation volumes than those obtained through tensile, creep or stress relaxation experiments, were obtained from the damping data. Furthermore, the activation volume was found to depend on the temperature as $\left(T_{\mathrm{c}}-T\right)^{-1}$, where $T_{\mathrm{c}}$ is close to the glass transition temperature, at the frequency of the measurements (of the order of $50 \mathrm{kHz}$ ). The amplitude independent damping increased continuously while the storage Young's modulus and the strain for the beginning of the amplitude dependence decreased continuously with the temperature, respectively.

Acknowledgements. We wish to express our appreciation to Dr. Élida B. Hermida for her helpful comments. This work was supported partially by the Consejo Nacional de Investigaciones Científicas y Técnicas (CONICET), the Antorchas Foundation, the University of Buenos Aires and the "Proyecto Multinacional de Tecnología de Materials" OAS-CNEA.

\section{REFERENCES}

1. (a) J. D. Ferry, "Viscoelastic Properties of Polymers," John Wiley, New York, N.Y., 1980, Chapter 12, p 279.

(b) J. D. Ferry, "Viscoelastic Properties of Polymers," John Wiley, New York, N.Y., 1980, Chapter 11.

2. G. E. Roberts and E. F. T. White, in "The Physics of Glassy Polymers," R. N. Haward, Ed., Applied Science Publishers, London, 1972, Chapter 3.

3. I. M. Ward, "Mechanical Properties of Solid Polymers," John Wiley \& Sons, Chichester, 1990, Chapter 7.

4. D. G. Gilbert, M. F. Ahshby, and P. W. R. Beaumont, J. Mater. Sci., 21, 3194 (1986).

5. C. Bauwens-Crowet, J. Mater. Sci., 8, 968 (1973).

6. A. I. Medalia, Rubber Chem. Technol., 51, 437 (1978).

7. G. E. Warnaka and H. T. Miller, Rubber Chem. Technol., 39, 1422 (1966).

8. W. P. Mason, "Physical Acoustics and the Properties of Solids," D. Van Nostrand, New York, N.Y., 1958.

9. J. Marx, Rev. Sci. Instrum., 22, 503 (1951).

10. W. H. Robinson and A. Edgar, IEEE Trans. Sonics and Ultras., Su-21, 98 (1974).

11. F. Povolo, A. J. Marzocca, and S. N. Goyanes, Polymer, 33, 2709 (1992).

12. F. Povolo and R. Gibala, Rev. Sci. Instrum., 40, 817 (1969).

13. F. Povolo and R. Gibala, Phil. Mag., 27, 1281 (1973).

14. F. Povolo, Scripa Metall., 8, 949 (1974).

15. H. Schafer and F. Povolo, Scripta Metall., 7, 445 (1973).

16. F. Povolo, Scripta Metall., 16, 885 (1982).

17. P. Peguin, J. Perez, and P. Gobin, Trans. AIME, 239, 438 (1967).

18. J. Heijboer, Kolloid Z., 148, 36 (1956).

19. W. Wunderlich, in "Polymer Handbook," J. Branrup and H. E. Immergut, Ed., John-Wiley \& Sons, New York, N.Y., 1976, p V-55.

20. E. Muzeau and J. Perez, Materials Science Forum, 119-121, 511 (1993).

21. J. Houssey, J. P. Cavrot, B. Escaig, and J. M. Lefebvre, J. Polym. Sci., Phys. Ed., 18, 311 (1980).

22. J. M. Lefebvre and B. Escaig, J. Mater. Sci., 20, 438 (1985).

23. B. E. Read, Polymer, 22, 1580 (1981).

24. D. W. Van Krevelen, "Properties of Polymers," Elsevier, Amsterdam, 1980, p 578. 


\section{F. Povolo and S. N. Goyanes}

25. R. N. Haward and G. Thackray, Proc. Roy. Soc. Ser. A, 302, 453 (1968).

26. A. S. Argon, Phil. Mag., 28, 839 (1973).

27. P. B. Bowden and S. Raha, Phil. Mag., 29, 149 (1974).

28. E. Pink, Mater. Sci. Eng., 22, 85 (1976).

29. J. R. White, J. Mater. Sci., 16, 3249 (1981).

30. B. Escaig, Poly. Eng. Sci., 24, 737 (1984).
31. J. Duxbury and I. M. Ward, J. Mater. Sci., 22, 1215 (1987).

32. J. P. Cavrot, J. Houssey, J. M. Lefebre, and B. Escaig, Mater. Sci. Eng., 36, 95 (1978).

33. F. Povolo and Élida B. Hermida, J. Appl. Polym. Sci., to be submitted. 